\title{
VSS CONTROL OF STRIP STEERING FOR HOT ROLLING MILLS
}

\author{
M.Okada, K.Murayama, Y.Anabuki, Y.Hayashi
}

West Japan Works (Kurashiki District), JFE Steel Corporation Kawasakidori 1-chome, Mizushima, Kurashiki 712-8511 Japan

\begin{abstract}
Strip steering is one of the most serious problems in the finishing mill in the hot strip rolling process. To improve steering control, a strip steering control system, which is based on sliding mode control and uses a steering observer, was designed and successfully applied to an actual plant. Copyright 2005 IFAC
\end{abstract}

Keywords: Variable-structure system, sliding-mode control, observer, steel industry, automatic control, metals

\section{INTRODUCTION}

In the hot strip rolling process in the steel industry, heated slabs are rolled to the required strip thickness and width. From the viewpoint of improved productivity and roll consumption, strip steering is one of the most serious problems in the finishing rolling process as it causes tail pinch. Parallel mill modulus control, which is one type of steering control, has been employed to prevent tail pinch. This method is based on proportional control to eliminate force differentials and achieve a levelling movement, but nevertheless, has certain inherent and practical disadvantages.

To improve steering control, a new steering control system based on sliding mode control using a steering observer was designed and successfully applied to an actual plant.

This paper first presents the concept of parallel mill modulus control. The new steering control system based on sliding mode control (SMC) using a steering observer is then described. Experimental results obtained with a commercial mill are also discussed.

\section{PROBLEMS OF CONVENTIONAL CONTROL}

Steering refers to lateral movement of the strip during rolling and is defined by the distance between the strip center and roll center. Fig. 1 shows an example of the steering phenomenon. In this case, a difference in the reduction ratio at the two sides of the strip occurs first, causing a difference in the entry strip velocity. The strip then deviates toward the side with the smaller reduction, and the roll gap increases on the side where the strip has moved. As a result, the difference in the reduction rate continues to increase, and the strip moves to the side with smaller

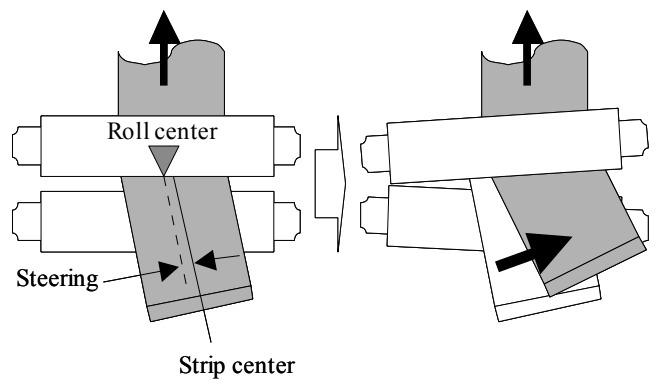

Fig. 1 Steering phenomenon 


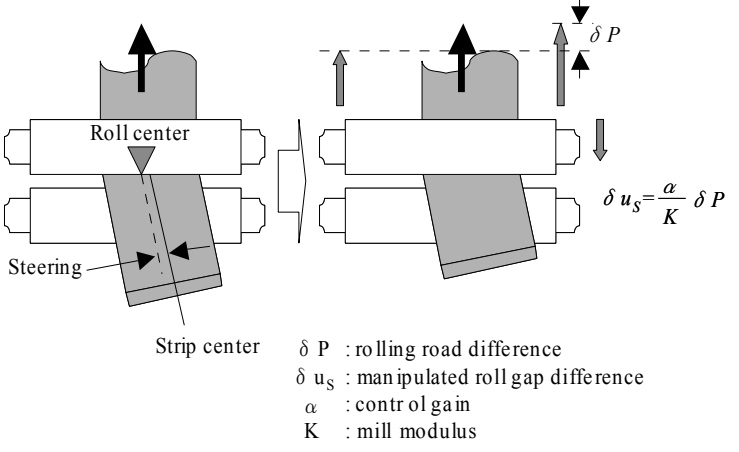

Fig. 2 Concept of paralle1 mill modulus control

reduction at an accelerating rate. Specifically, if steering once occurs, its rate of increases is approximately proportional to the second power.

Parallel mill modulus control is a conventional control method proposed by Kimura et al.(1983). Fig. 2 shows the concept. The increase in the roll gap caused by the difference in the rolling load is cancelled, preventing further increases in the difference in the reduction ratio at the two sides. However, due to the initial difference in the reduction ratio, the strip continues to move to the side with smaller reduction. Moreover, as practical disadvantages, this method requires high response and large movement of the actuator, and the large movement can cause tears in the strip tail end. An approach based on simple P-control using a steering sensor with no delay was also proposed by Kuwano et al. (1986), but in order to detect steering without delay, the sensor must be installed in a harsh environment, making it difficult to maintain adequate accuracy. A state feedback compensator using a state observer was proposed by Okamura et al. (1996). However, in hot rolling processes, the delay in the state observer cannot be ignored.

\section{DEVELOPMENT OF NEW CONTROL METHOD}

\subsection{Concept of sliding mode control}

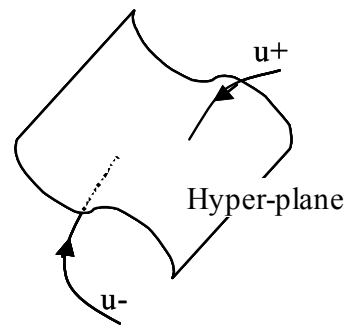

Fig. 3 Concept of sliding mode control

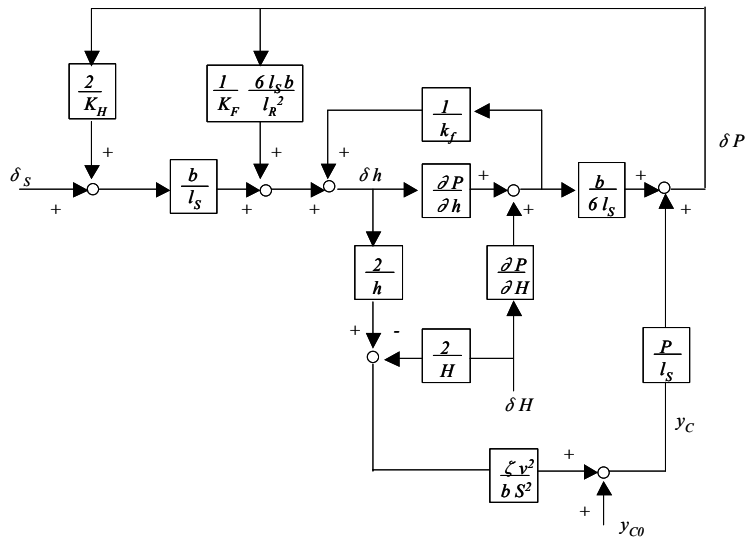

Fig. 4 Block diagram of steering growing model

Fig.3 shows the concept of sliding mode control. Sliding mode control is a type of variable-structure control (VSS), which causes system states to converge on a predefined hyper-plane by frequent switching of the system structure. Due to the sliding motion, it is robust with respect to parameter uncertainty and external disturbances (Utkin, 1977) (DeCarlo et al., 1988).

\subsection{Modelling of steering}

A steering growing model was discussed by Nakajima et al. (1980). Fig. 4 shows a block diagram of the steering model, where $y_{C}$ is strip steering, $y_{C 0}$ is initial strip steering, $\zeta$ is a constant which depends on rolling conditions, $v$ is the entry strip velocity, $S$ is the Laplacian operator, $P$ is the total rolling load, $b$ is the strip width, $l_{S}$ is the distance between bearings, $l_{R}$ is the roll face length, $h$ is the exit strip thickness, $H$ is the entry strip thickness, $K_{H}$ is the mill housing modulus, $K_{F}$ is the modulus between the work roll and the backup roll, $k_{f}$ is the modulus between the work roll and the strip in the roll bite, $\delta h$ is the exit strip wedge, $\delta H$ is the entry strip wedge, $\delta P$ is the rolling load difference, and $\delta_{S}$ is the roll gap difference. From the block diagram, the following three equations are obtained:

$$
\begin{aligned}
& \delta h=\frac{1}{k_{f}}\left(\frac{\partial P}{\partial h} \delta h+\frac{\partial P}{\partial H} \delta H\right)+\frac{1}{K_{F}} \frac{6 l_{s} b}{l_{R}{ }^{2}} \delta b+\frac{b}{l_{s}}\left(\frac{2}{K_{H}} \delta P+\delta_{S}\right), \\
& \delta P=\frac{b}{6 l_{s}}\left(\frac{\partial P}{\partial h} \delta h+\frac{\partial P}{\partial H} \delta H\right)+\frac{P}{l_{s}} y_{C}, \\
& \ddot{y}_{C}=\frac{\zeta v^{2}}{b}\left(\frac{2}{h} \delta h-\frac{2}{H} \delta H\right) .
\end{aligned}
$$

From Eq. (1) and (2),

$$
\delta h=\frac{Q_{\text {in }}}{K_{1}+Q_{\text {out }}} \delta H+\frac{6 P}{b} \frac{1}{\left(K_{1}+Q_{\text {out }}\right)} \frac{K_{1}}{K_{2}} y_{C}+\frac{b}{l_{s}} \frac{K_{1}}{K_{1}+Q_{\text {out }}} \delta_{S},
$$


where,

$Q_{\text {in }}=\frac{\partial P}{\partial H}$,

$Q_{\text {out }}=-\frac{\partial P}{\partial h}$,

$\frac{1}{K_{1}}=\frac{1}{k_{f}}+\frac{1}{K_{F}} \frac{b^{2}}{l_{R}^{2}}+\frac{1}{3} \frac{1}{K_{H}} \frac{b^{2}}{l_{s}^{2}}$,

$\frac{1}{K_{2}}=\frac{1}{K_{F}} \frac{b^{2}}{l_{R}^{2}}+\frac{1}{3} \frac{1}{K_{H}} \frac{b^{2}}{l_{s}^{2}}$

Next, from Eq. (3) and (4),

$\ddot{y}_{C}=\frac{12 P \zeta v^{2}}{b^{2} h} \frac{1}{K_{1}+Q_{\text {out }}} \frac{K_{1}}{K_{2}} y_{C}+\frac{2 \zeta v^{2}}{h l_{s}} \frac{K_{1}}{K_{1}+Q_{\text {out }}} \delta_{S}+d$,

where,

$d=\frac{2 \zeta v^{2}}{b}\left(\frac{1}{h} \frac{Q_{\text {in }}}{K_{1}+Q_{\text {out }}}-\frac{1}{H}\right) \delta H+\ddot{y}_{C 0}$.

Here, $d$ can be handled as disturbance, because $\delta H$ and $y_{C 0}$ are exogenous inputs.

The model of the hydraulic gap control device is expressed as

$$
\delta_{S}=\frac{1}{1+T_{s} s} \delta u_{S}+\delta_{S 0},
$$

where, $T_{S}$ is the time constant of the gap control device, $\delta u_{S}$ is the input to the device, and $\delta_{S O}$ is the initial roll gap difference.

Therefore, the state space equation of the plant can be expressed as follows:

$\dot{\mathbf{x}}=\mathbf{A x}+\mathbf{B} \delta u_{S}+\mathbf{E d}$,

$y_{C}=\mathbf{C x}$,

where,

$$
\begin{aligned}
& \mathbf{x}=\left[\begin{array}{lll}
y_{C} & \dot{y}_{C} & \delta_{S}
\end{array}\right]^{T}, \\
& \mathbf{d}=\left[\begin{array}{llll}
\delta H & \ddot{y}_{C 0} & \delta_{S 0} & \dot{\delta}_{S 0}
\end{array}\right]^{T}, \\
& \mathbf{A}=\left[\begin{array}{ccc}
0 & 1 & 0 \\
a_{21} & 0 & a_{23} \\
0 & 0 & -\frac{1}{T_{S}}
\end{array}\right], \\
& \mathbf{B}=\left[\begin{array}{lll}
0 & 0 & \frac{1}{T_{S}}
\end{array}\right]^{T}, \\
& \mathbf{C}=\left[\begin{array}{lll}
1 & 0 & 0
\end{array}\right] \text {, } \\
& \mathbf{E}=\left[\begin{array}{cccc}
0 & 0 & 0 & 0 \\
e_{21} & 1 & 0 & 0 \\
0 & 0 & \frac{1}{T_{S}} & 1
\end{array}\right],
\end{aligned}
$$

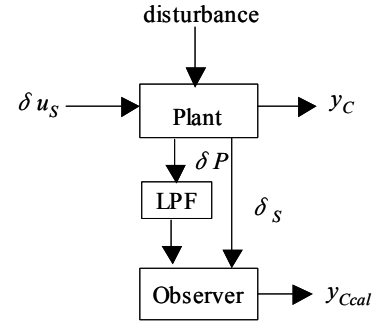

Fig. 5 Block diagram of steering observer

$$
\begin{aligned}
& a_{21}=\frac{12 P \zeta v^{2}}{b^{2} h} \frac{1}{K_{1}+Q_{\text {out }}} \frac{K_{1}}{K_{2}} \\
& a_{23}=\frac{2 \zeta v^{2}}{h l_{s}} \frac{K_{1}}{K_{1}+Q_{\text {out }}} \\
& e_{21}=\frac{2 \zeta v^{2}}{b}\left(\frac{1}{h} \frac{Q_{\text {in }}}{K_{1}+Q_{\text {out }}}-\frac{1}{H}\right) .
\end{aligned}
$$

\subsection{Design of steering observer}

To obtain response, steering should be calculated directly. From Eq. (2) and (4),

$$
\begin{aligned}
y_{C} & =\frac{l_{S}}{P} \frac{K_{1} K_{2}+K_{2} Q_{\text {out }}}{K_{1} K_{2}+\left(K_{2}-K_{1}\right) Q_{\text {out }}} \delta P \\
& +\frac{b^{2}}{6 l_{S} P} \frac{K_{2}^{2} Q_{\text {out }}}{K_{1} K_{2}+\left(K_{2}-K_{1}\right) Q_{\text {out }}} \delta_{S}+d_{H} \\
d_{H} & =-\frac{b}{6 P} \frac{K_{1} K_{2} Q_{\text {in }}}{K_{1} K_{2}+\left(K_{2}-K_{1}\right) Q_{\text {out }}} \delta H,
\end{aligned}
$$

Because $\delta H$ is an exogenous input, $d_{H}$ is ignored to simplify the observer. In the actual plant, because the output of the load sensor includes some noise factors, a low-pass filter (LPF) is installed, as shown in Fig.5.

Fig.6 shows the results of a simulation which was performed to confirm the appropriateness of ignoring $\delta H$ and installing the LPF. The results showed that ignoring $\delta H$ and using an LPF is a reasonable method of observing steering.

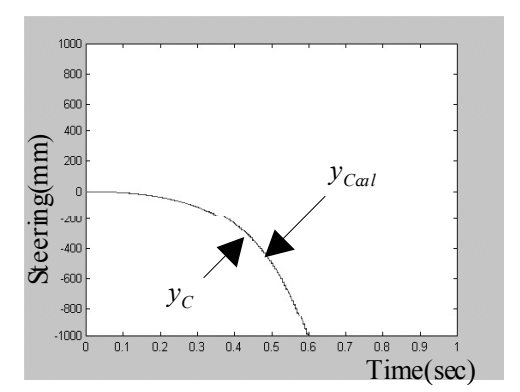

Fig. 6 Simu lation result of steering observer 


\subsection{Design strategy of sliding sector}

Because the response of the hydraulic roll gap device is sufficiently high in general hot rolling processes, the lag of the hydraulic gap control device can be ignored in controller design. Therefore, the state space equations used in designing the controller were as follows:

$\dot{\mathbf{x}}=\mathbf{A x}+\mathbf{B} \delta u_{S}$,

$y_{C}=\mathbf{C x}$,

where,

$\mathbf{x}=\left[\begin{array}{ll}y_{C} & \dot{y}_{C}\end{array}\right]^{T}$,

$\mathbf{A}=\left[\begin{array}{cc}0 & 1 \\ a_{21} & 0\end{array}\right]$,

$\mathbf{B}=\left[\begin{array}{ll}0 & a_{23}\end{array}\right]^{T}$,

$\mathbf{C}=\left[\begin{array}{ll}1 & 0\end{array}\right]$,

$a_{21}=\frac{12 P \zeta v^{2}}{b^{2} h} \frac{1}{K_{1}+Q_{o u t}} \frac{K_{1}}{K_{2}}$,

$a_{23}=\frac{2 \zeta v^{2}}{h l_{s}} \frac{K_{1}}{K_{1}+Q_{\text {out }}}$.

The sliding sector $\sigma$ is defined as

$\sigma=\mathbf{S x}$

$\mathbf{S}=\left[\begin{array}{ll}c & 1\end{array}\right]$

A sliding sector design strategy based on pole assignment was proposed by C. A. Woodham et al. (1993). When system states are converging on the sector, they satisfy

$\mathbf{S x}=0$.

Substituting (21) into (16), the pole of the system becomes

$p=-\frac{a_{21}}{c}$,

where, $p$ is the system pole. From the above, any pole $p$ can be obtained by choosing parameter $c$.

\subsection{Design of VSS controller}

A VSS controller, which converges the steering states on the sector, was introduced. This method is based on the eventual sliding mode switching scheme (J. Y. Hung, 1993). State space equations (16)-(20) are considered in the design of the VSS controller.

The input $\delta u_{S}$ is assumed to be

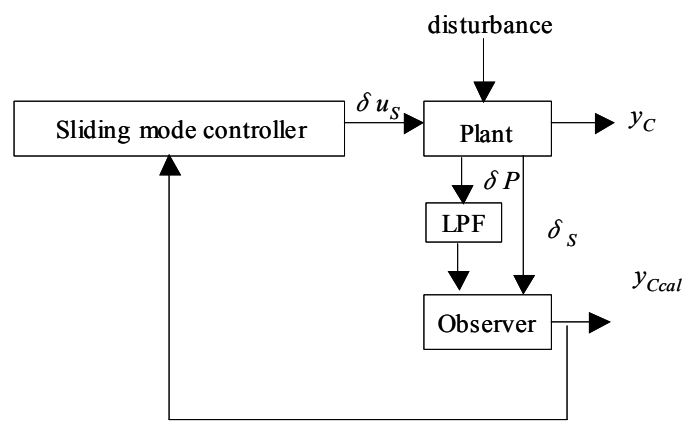

Fig. 7 Block diagram of steering control system

$\delta u_{S}=-(\mathbf{S B})^{-1} \mathbf{S A x}-k \frac{\sigma}{|\sigma|}$,

where, $k$ is control gain. To converge on the sector, $\sigma$ should converge to zero. Therefore, the following Lyapunov function for $\sigma$ is considered:

$V=\frac{\sigma^{2}}{2}$.

The derivative of the Lyapunov function is

$$
\begin{aligned}
\dot{V} & =\sigma^{2} \\
& =\sigma \mathbf{S A x}+\sigma \mathbf{S B} \delta u_{S} .
\end{aligned}
$$

Substituting (23) into (25), the condition of the existing sliding mode is

$\dot{V}=-k \mathbf{S B} \frac{\sigma^{2}}{|\sigma|}<0$,

i.e.,

$k>0$,

because, from equations (18) and (20),

$\mathbf{S B}=a_{23}>0$.

The VSS control system must frequently switch the system structure, as follows:

$\delta u_{S}=\left\{\begin{array}{ll}-(\mathbf{S B})^{-1} \mathbf{S A x}-k & \sigma>0 \\ -(\mathbf{S B})^{-1} \mathbf{S A x}+k & \sigma<0\end{array}\right.$.

Because the switching frequency should theoretically be infinite, inherent chattering phenomena exist in VSS control systems due to the actual finite switching frequency or switching delay. To reduce these chattering phenomena, a saturated function $\sigma /$ $(|\sigma|+\delta)$ is substituted for $\sigma /|\sigma|$ Therefore, the input $\delta u_{S}$ is derived as follows: 
(a) $y_{C O}$ disturbance: $+10[\mathrm{~mm}]$

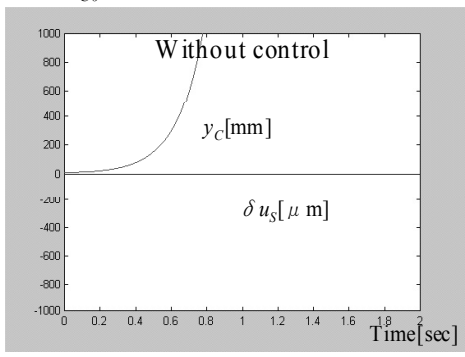

(b) $\delta H$ disturbance: $+0.1[\mathrm{~mm}]$

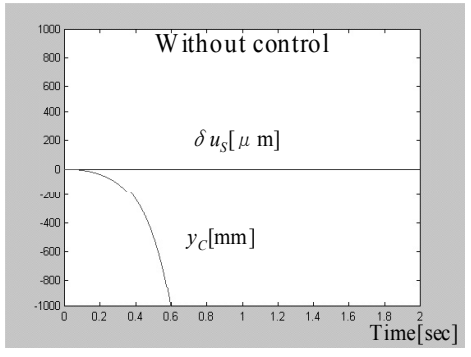

(c) $\delta_{\mathrm{S}}$ disturbance: $+0.1[\mathrm{~mm}]$

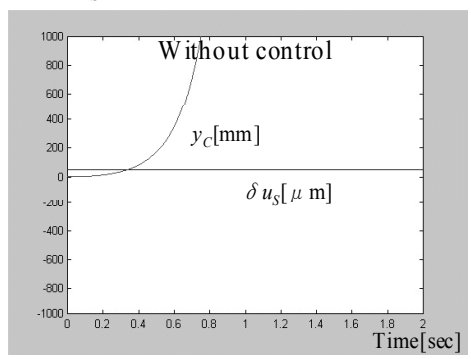

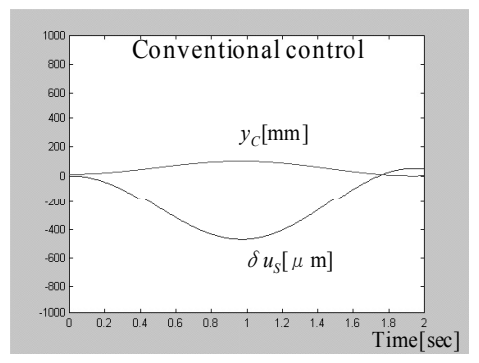
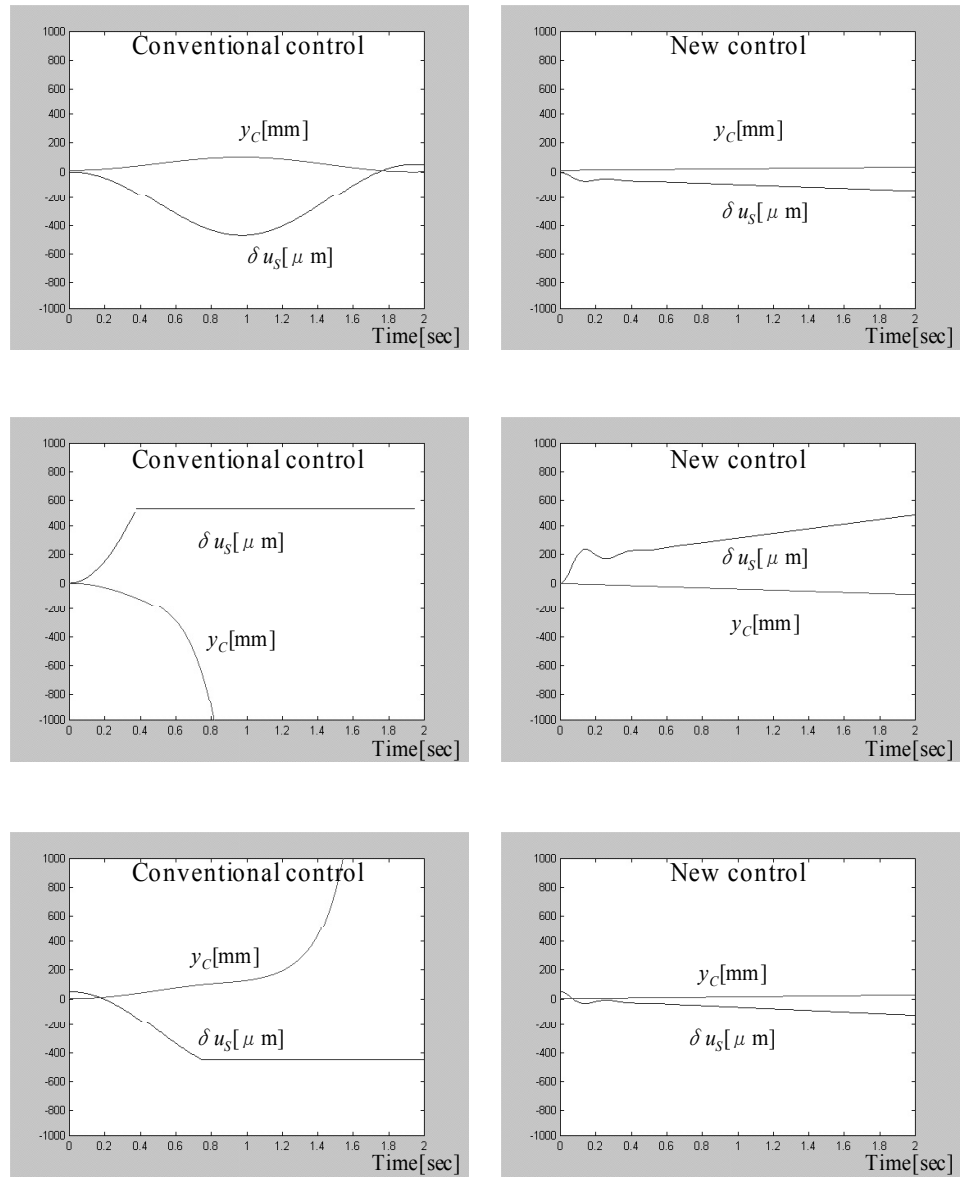

Fig. 8 Simu lation results ( Low carbon, $3.0 \mathrm{~mm} \times 1030 \mathrm{~mm}$ )

$$
\begin{aligned}
\delta u_{S} & =-(\mathbf{S B})^{-1} \mathbf{S A x}-k \frac{\sigma}{|\sigma|+\delta} \\
& =-\frac{a_{21}}{a_{23}} y_{C}-\frac{c}{a_{23}} \dot{y}_{C}-k \frac{\sigma}{|\sigma|+\delta}
\end{aligned}
$$

Fig. 7 shows the block diagram of the steering control system.

\section{RESULTS}

\subsection{Simulation results}

The results of a simulation for comparison with conventional control are shown in Fig. 8. Here, it was assumed that the disturbances were the initial strip steering $\left(y_{C O}=10 \mathrm{~mm}\right)$, the entry strip wedge ( $\delta$ $H=0.1 \mathrm{~mm})$, and the roll gap difference ( $\delta_{S}=0.1 \mathrm{~mm}$ ). The simulation was performed based on the condition that the material is low carbon steel, the strip dimensions are $3.0 \mathrm{~mm} * 1030 \mathrm{~mm}$, the gains in conventional control are the same as those actually used, and the system pole is $s=1$.
The simulation results showed that, when the new control method is compared with conventional control, steering deviation is reduced and the control system is more stable. Furthermore, the system input $\delta \mathrm{u}_{\mathrm{s}}$ is also reduced by early output from the controller, and chattering phenomena do not occur.

\subsection{Practical results}

The new steering control system was applied to the tail end of strip in the finishing mill at West Japan Works (Kurashiki District). The experimental results obtained with this commercial mill are shown in Fig. 9, Fig. 10, and Fig.11. In this case, the control gains are the same as those in the simulation, and the material is low carbon steel $(3.0 \mathrm{~mm} * 1000 \mathrm{~mm})$ from the same lot. Fig. 9 and Fig. 10 show examples of conventional control results and results with the new control method, respectively. From these results, steering deviation and the rolling load difference are reduced, and the system input is also reduced, as in the simulation results.

Fig.11 shows the average of the absolute values of steering in each case. The new steering control 
method is more stable, and strip steering has been improved by more than $50 \%$.

\section{CONCLUSION}

This paper described a newly-developed VSS control system for strip steering in hot rolling mills, which is based on sliding mode control using a steering observer. The experimental results show that the new control system is superior to the conventional system.

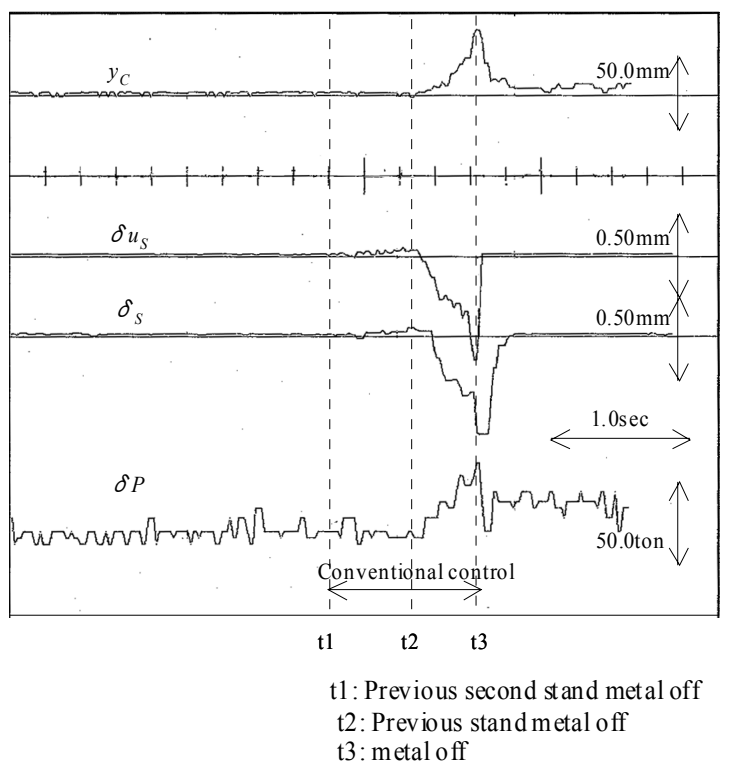

Fig. 9 Examp le of experimental results using the conventional control ( Low carbon, $3.0 \mathrm{~mm} \times 1000 \mathrm{~mm}$ )

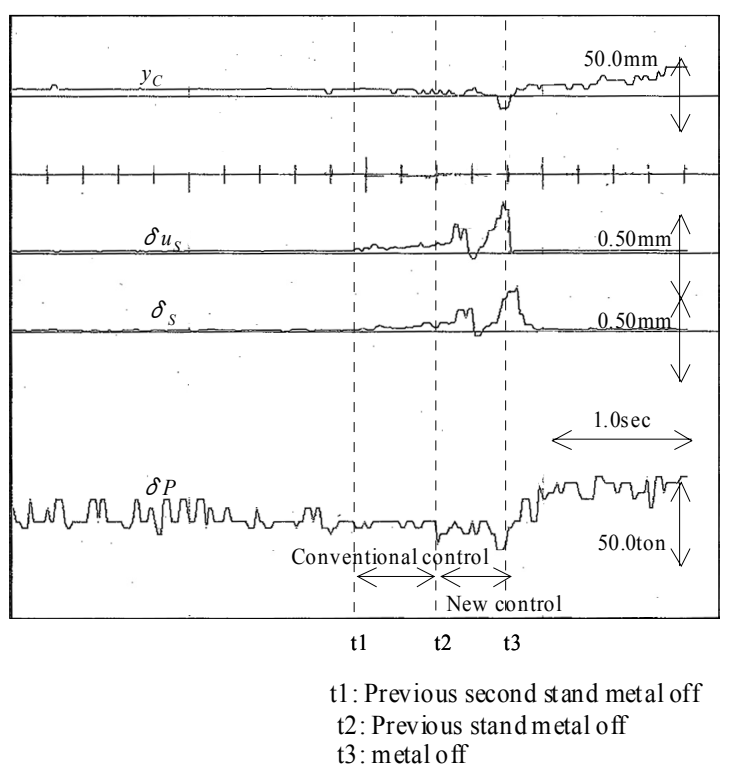

Fig. 10 Example of experimental results using the new control ( Low carbon, $3.0 \mathrm{~mm} \times 1000 \mathrm{~mm}$ )

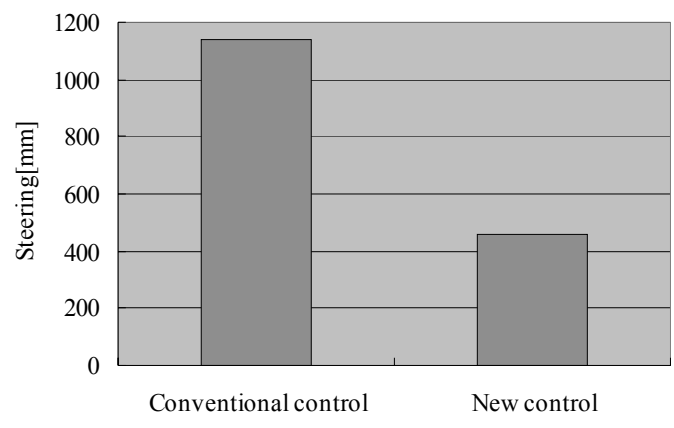

Fig. 11 Average of absolute values of steering ( Low carbon, $3.0 \mathrm{~mm} \times 1000 \mathrm{~mm}, \mathrm{n}=20$ )

\section{REFERENCES}

Decarlo, R. A., Zak, S. H. and Matthew, G. P. (1988). Variable structure control of nonlinear multi-variable system, Proceedings of IEEE, 76-3, 212-232.

Hung, J. Y. (1993). Variable structure control: A survey, IEEE Trans. Ind. Electron, 40, 2-22.

Kimura, T. and Tagawa. M. (1983). Automatic steering control of strip rolling mills, Hitachi Review, 65-2, 25-30.

Kuwano, H. and Takahashi, N. (1986). Sensor-type automatic steering control system for rolling mill $1^{\text {st }}$ report-, Ishikawajima-Harima Engineering Review, 26-1, 35-40.

Nakajima, K., Kajiwara, T., Kimura, T. , Kikuma, T. , Matsumoto, H. and Tagawa, M. (1980). Automatic side-walk control in hot strip mill, The 1980 Japanese Spring Conf. For the Technology of Plasticity, 116, 61-64.

Okamura, Y. and Hoshino, I. (1996). State feedback control of the strip steering for aluminum hot rolling mills, $13^{T H}$ World Congress of IFAC, 7b-03, 481-485.

Utkin, V. I. (1977). Variable structure systems with sliding mode, IEEE Trans. Automat. Contr., 22-4, 212-222.

Woodham, C. A. and Zinober, A. S .I. (1993), Int .J. Control, 57-5,1021-1037. 